\title{
Poster Number: P 0991 \\ Maternal nutritional status during early pregnancy and its consequences on newborns at birth
}

A. Hussain ${ }^{1}$, B. Bhowmik ${ }^{1}$, T. Siddiquee ${ }^{1}$, H. lasmin ${ }^{1}$, G.A. Hitman 2 , A.A. Khan ${ }^{1}$

${ }^{1}$ Diabetic Association of Bangladesh, Centre for Global Health Research, Dhaka, Bangladesh.

${ }^{2}$ Queen Mary University of London, Blizard Institute- Barts and The London School of Medicine and Dentistry, London E1 2AT, Bangladesh

\section{Background}

The supply of nutrients to the fetus is the major influence that regulate its growth.

Aim

To investigate the maternal nutritional status including micro-nutrients during early pregnancy and its impact on newborns at birth.

Method

- A total of 498 women $\geq 18$ years participating in a prospective study conducted in 5 different areas of Dhaka, Bangladesh were studied.

- Women were recruited randomly during first antenatal visit between April 2011 to June 2012.

- Inclusion criteria:

- Expectant mothers in 1st trimester of pregnancy.

- Singleton primi gravida pregnancy.

- Conception without treatment of infertility.

- Willing to join the study

- Exclusion Criteria:

- History of gestational diabetes (GDM), congenital malformations and macrosomia in previous pregnancies.

- Population characteristics (mother and infant), in the form of anthropometry, blood tests, and health, lifestyle and dietary habits were analyzed at different points throughout the study.

- Linear regression was used to investigate the association of interest.

Results

- Among the study participants, $30.9 \%$ were underweight $\left(<18.5 \mathrm{~kg} / \mathrm{m}^{2}\right)$, $48.4 \%$ had normal weight $\left(18.5-22.9 \mathrm{~kg} / \mathrm{m}^{2}\right)$, and $20.7 \%$ were overweight $\left(\geq 23.0 \mathrm{~kg} / \mathrm{m}^{2}\right)$ (Figure 1).

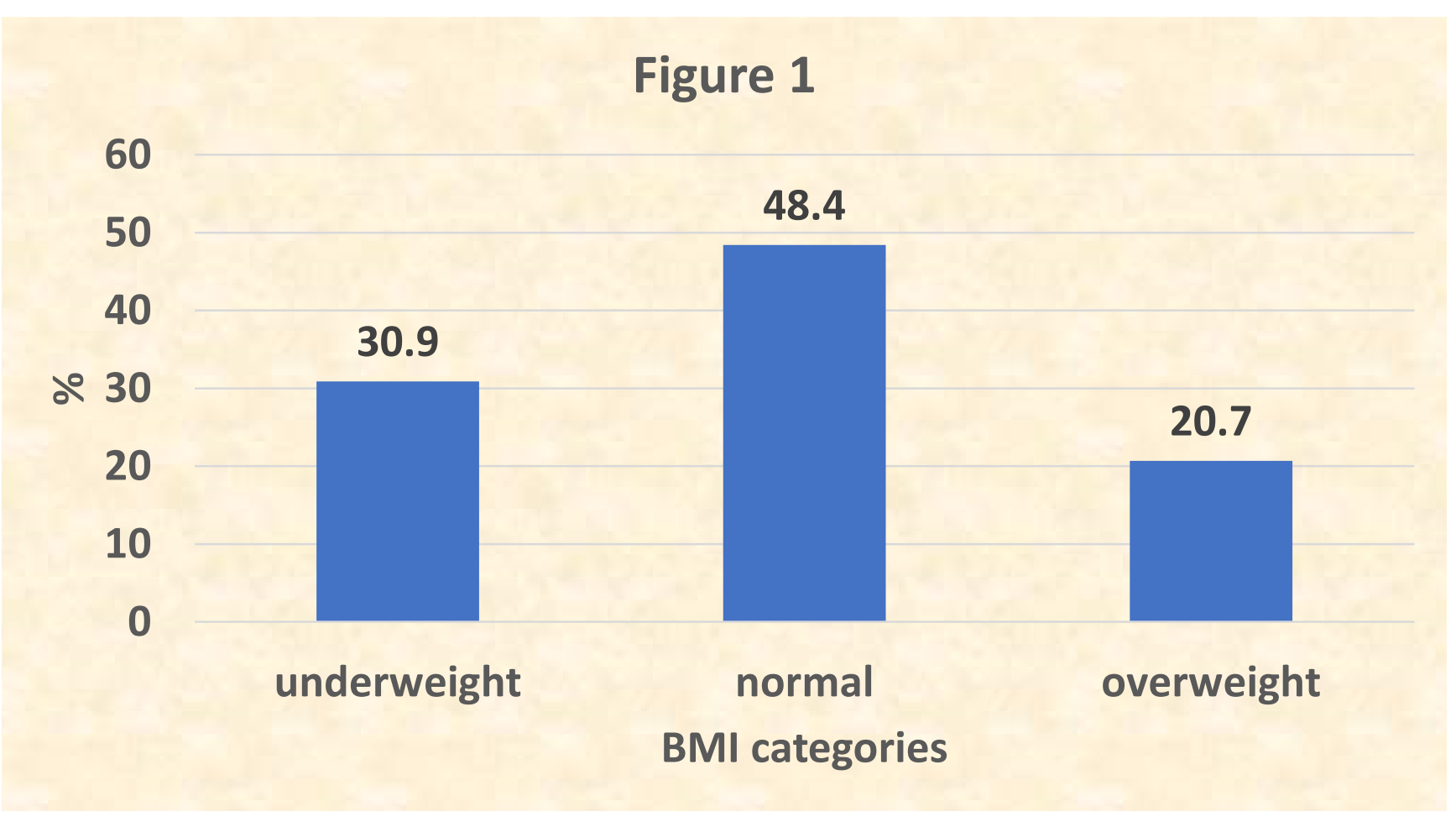

- The prevalence of anemia $(\mathrm{Hb},<11 \mathrm{~g} / \mathrm{dl})$, vitamin $D$ deficiency $(<30 \mathrm{nmol} / \mathrm{l})$, vitamin B12 deficiency $(<200 \mathrm{pg} / \mathrm{ml})$, high homocysteine level $(>15 \mu \mathrm{mol} / \mathrm{l})$, folate deficiency $(<3 \mathrm{ng} / \mathrm{ml})$ and ferritin deficiency $(<13 \mathrm{ng} / \mathrm{ml})$ were $19.5 \%$, $46.4 \%, 15.1 \%, 1.2 \%, 0.4 \%$, and $12.7 \%$ respectively. Hyperglycemia in pregnancy was $31.5 \%$ (Figure 2).

\section{Reference}

1. Victora CG, Adair L, Fall C, et al, the Maternal and Child Undernutrition Study Group: Maternal and child undernutrition: consequences for adult health and human capital. Lancet 2008; 371: 340-357.
Figure 2

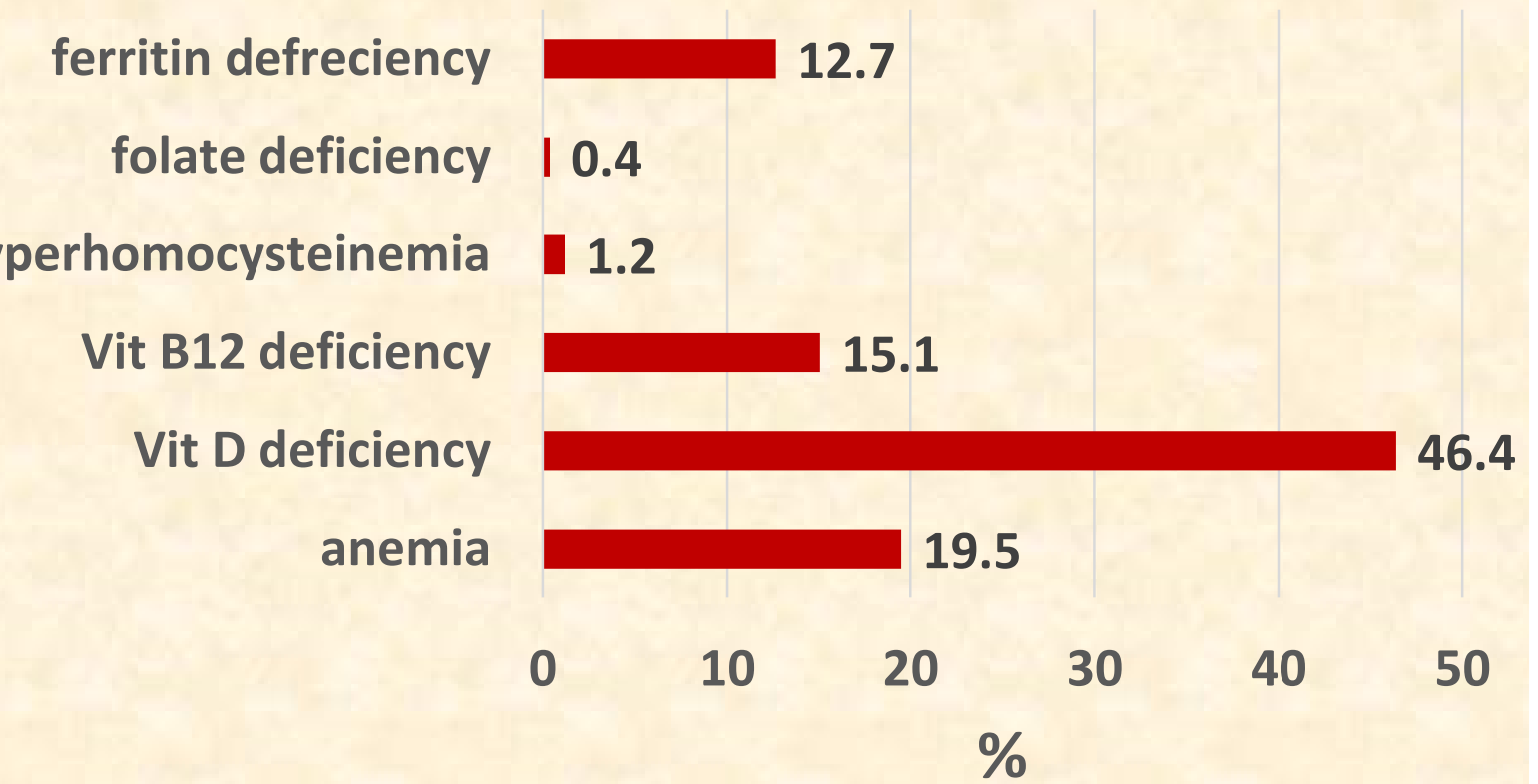

- Incidence of low birth weight ( $<2500 \mathrm{~g}$ ) and preterm delivery ( $<37$ week) were $11.4 \%$ and $5 \%$ respectively (Figure 3 ).

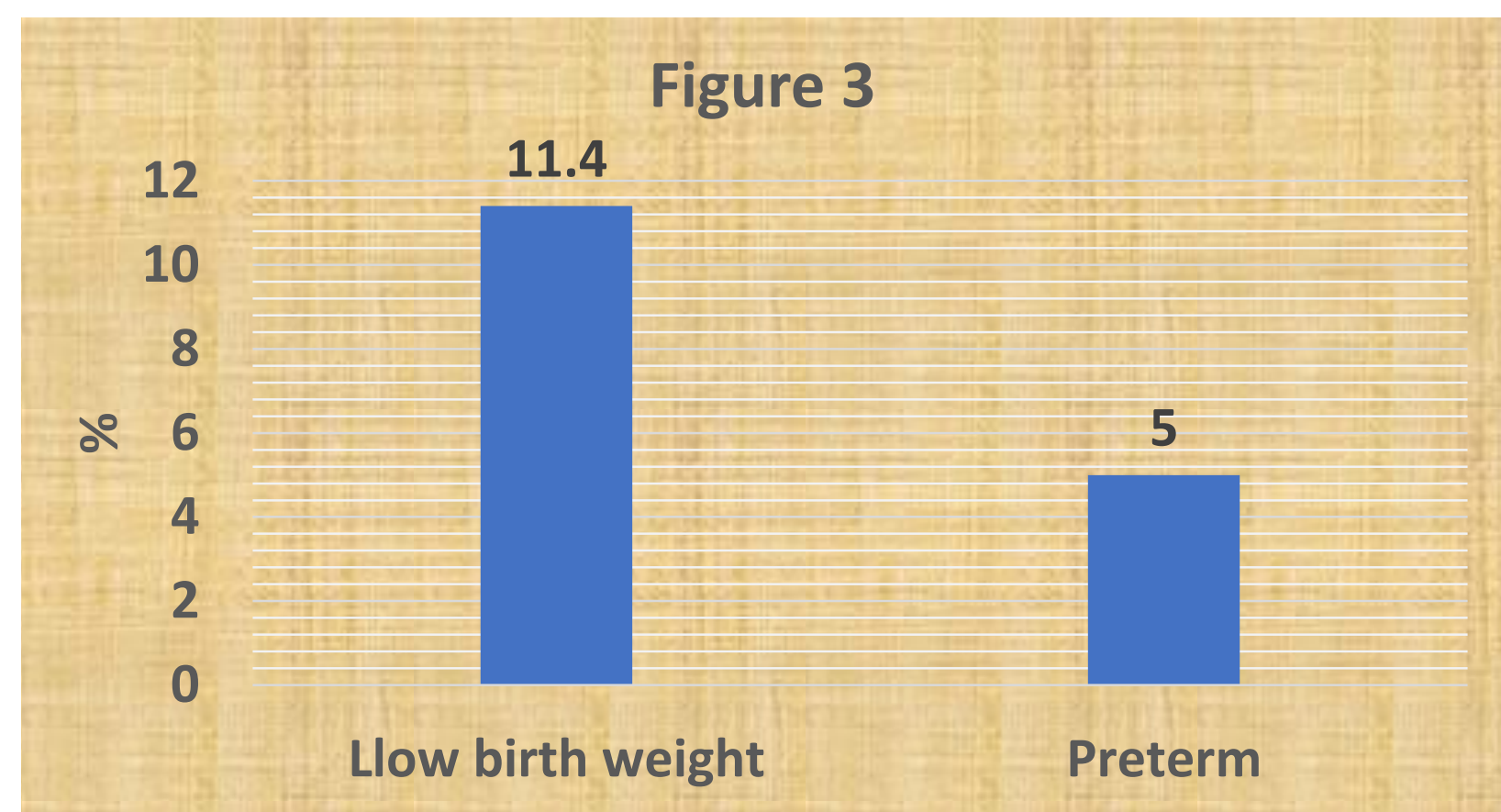

- Maternal height was positively ( $(14.97, p=0.02$ ) and homocysteine was negatively ( $B-33.26, p=0.02$ ) associated with neonatal birth weight. In addition, folate level in cord blood was positively ( $(5.21, p=0.03$ ) and triglyceride was negatively ( $(-2.96, p=0.03$ ) associated with neonatal birth weight.

\begin{tabular}{|c|c|c|c|c|c|c|}
\hline \multirow[b]{2}{*}{$\begin{array}{l}\text { Maternal } \\
\text { variables }\end{array}$} & \multicolumn{2}{|c|}{$\begin{array}{l}{ }^{1} \text { Full model } \\
\text { AIC }=14.92\end{array}$} & \multicolumn{2}{|c|}{$\begin{array}{c}{ }^{2} \text { Model } 2 \\
\text { AIC }=14.76\end{array}$} & \multicolumn{2}{|l|}{$\begin{array}{c}{ }^{3} \text { Model } 3 \\
\text { AIC }=14.78\end{array}$} \\
\hline & $\beta(95 \% \mathrm{Cl})$ & P-value & $\beta(95 \% \mathrm{Cl})$ & P-value & $\beta(95 \% \mathrm{Cl})$ & $\begin{array}{c}P \text { - } \\
\text { value }\end{array}$ \\
\hline Height $(\mathrm{cm})$ & $114.78(1.06,28.49)$ & 0.04 & $14.97(2.51,27.43)$ & 0.02 & $13.58(1.40,25.75)$ & 0.03 \\
\hline $\begin{array}{l}\text { Homocysteine } \\
(\mu \mathrm{mol} / \mathrm{l})\end{array}$ & $-35.58(-68.07,-3.08)$ & 0.03 & $-33.26(-60.21,-6.31)$ & 0.02 & $-29.41(-56.23,-2.58)$ & 0.03 \\
\hline
\end{tabular}

${ }^{3}$ Model 3 was fitted by selecting variables with $P \leq 0.05$ from the univariate analysis Using the Akaike information criteria (AIC).

\begin{tabular}{|l|c|c|c|c|c|c|}
\hline & \multicolumn{2}{|c|}{${ }^{1}$ Full Model } & \multicolumn{2}{c|}{${ }^{2}$ Model 2 } & \multicolumn{2}{c|}{${ }^{3}$ Model 3} \\
AIC $=14.76$ & AIC $=14.73$ & \multicolumn{2}{c|}{ AIC $=14.75$} \\
\hline Neonatal variables & $\beta(95 \% \mathrm{Cl})$ & P-value & $\beta(95 \% \mathrm{Cl})$ & $\begin{array}{c}\text { P- } \\
\text { value }\end{array}$ & $\beta(95 \% \mathrm{Cl})$ & P-value \\
\hline Triglyceride $(\mathrm{mg} / \mathrm{dll})$ & $-2.48(-5.53,0.58)$ & 0.11 & $-2.96(-5.47,-0.45)$ & 0.02 & $\begin{array}{c}-3.35(-5.84,- \\
0.85)\end{array}$ & 0.01 \\
\hline Folate $(\mathrm{ng} / \mathrm{ml})$ & $5.17(0.03,10.31)$ & 0.05 & $5.21(0.41,10.01)$ & 0.03 & $4.68(0.37,9.00)$ & 0.03 \\
\hline
\end{tabular}

Model is adjusted for all neonatal variables

Discussion

- Maternal malnutrition including both macro and micronutrients problem during early pregnancy were prevalent in our study participants.

- Maternal height and homocysteine in early pregnancy were the independent predictors of neonatal birth weight. In addition, triglyceride and folate levels in cord blood were also significantly associated with newborns birth weight.

Conflict of Interest

No potential conflict of interest

Funded by: European Union (EU-7)

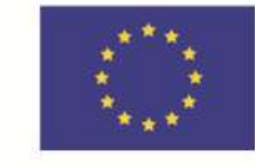

